\title{
Exogenous stimulation with Eclipta alba promotes hair matrix keratinocyte proliferation and downregulates TGF- $\beta 1$ expression in nude mice
}

\author{
SHAHNAZ BEGUM ${ }^{1}$, MI RA LEE ${ }^{1}$, LI JUAN GU ${ }^{1}$, JAMIL HOSSAIN ${ }^{2}$ and CHANG KEUN SUNG ${ }^{1}$ \\ ${ }^{1}$ Department of Food Science and Technology, College of Agriculture and Biotechnology, \\ ${ }^{2}$ Molecular Genetics and Genomics Laboratory, Department of Horticulture, \\ Chungnam National University, Daejeon 305-764, Republic of Korea
}

Received August 28, 2014; Accepted October 7, 2014

DOI: $10.3892 / \mathrm{ijmm} .2014 .2022$

\begin{abstract}
Eclipta alba (L.) Hassk (E. alba) is a traditionally acclaimed medicinal herb used for the promotion of hair growth. However, to the best of our knowledge, no report has been issued to date on its effects on genetically distorted hair follicles (HFs). In this study, we aimed to identify an agent (stimuli) that may be beneficial for the restoration of human hair loss and which may be used as an alternative to synthetic drugs. We investigated the effects of petroleum ether extract (PEE) and different solvent fractions of E. alba on HFs of nude mice. Treatment was performed by topical application on the backs of nude mice and the changes in hair growth patterns were evaluated. Histological analysis was carried out to evaluate the HF morphology and the structural differences. Immunohistochemical (IHC) staining was performed to visualize follicular keratinocyte proliferation. The histological assessments revealed that the PEE-treated skin specimens exhibited prominent follicular hypertrophy. Subsequently, IHC staining revealed a significant increase $(p<0.001)$ in the number of follicular keratinocytes in basal epidermal and matrix cells. Our results also demonstrated that PEE significantly $(\mathrm{p}<0.001)$ reduced the levels of transforming growth factor- $\beta 1$ (TGF- $\beta 1$ ) expression during early anagen and anagen-catagen transition. Our results suggest that PEE of E. alba acts as an important exogenous mediator that stimulates follicular keratinocyte proliferation and delays terminal differentiation by downregulating TGF- $\beta 1$ expression. Thus, this study highlights the potential use of PEE of E. alba in the treatment of certain types of alopecia.
\end{abstract}

Correspondence to: Dr Chang Keun Sung, Department of Food Science and Technology, College of Agriculture and Biotechnology, Chungnam National University, 99, Daehak-Ro, Daejeon 305-764, Republic of Korea

E-mail: sungck80@gmail.com

Key words: Eclipta alba, nude mice, hair follicle, keratinocyte, $\mathrm{BrdU}$, transforming growth factor- $\beta 1$

\section{Introduction}

The hair follicle (HF) is the most prominent mini organ of the skin and is remarkable for its dynamic structure. The fundamental characteristic of hair biology involves the production of a hair shaft (anagen), apoptosis driven by regression (catagen), and the relative resting (telogen) phase. The HF undergoes repeated cycles of regression and regeneration throughout the lifetime of an organism. Each phase of the hair cycle is characterized by the distinctive, strictly co-coordinated progression of tissue proliferation, differentiation and apoptosis, thus maintaining hairy phenotype of an organism $(1,2)$.

The growth and development of HFs are activated by a variety of growth factors, hormones and cytokines on different hair growth phases. Molecules, such as fibroblast growth factor 5 (FGF5), brain-derived neurotrophic factor (BDNF), p75, p53 and transforming growth factor (TGF)- $\beta 1$ promote the induction of the catagen phase (3). Among these molecules, the overexpression of TGF- $\beta 1$ in the epidermis of transgenic mice has been shown to lead to the inhibition of normal skin development (4). Therefore, recently, TGF- $\beta 1$ has been reported to control murine HF regression (catagen) in vivo (5).

The most striking characteristic of nude mice is the complete lack of fur development; these mice have been established as a valuable biomedical tool since their discovery in 1966 (6). Although the nude mouse phenotype appears hairless at the skin, its dermis contains a substantial number of active HFs. However, these follicles are aberrant and undeveloped $(6,7)$. The impaired differentiation of nude follicles exhibits structural imperfections of the cortex, hair cuticle and inner root sheath (8). As a result, a hair shaft bend and coil at the sebaceous gland and failure to penetrate the epidermis, is responsible for the lack of external fur coat in nude mice $(9,10)$.

Various research groups have used nude mice as a model for hair biology and have reported that cyclosporin A (CsA) (11), keratinocyte growth factor (KGF) (12) and AS101 (13) are potential therapeutic tools. However, chemically synthesized drugs are known for their adverse side-effects. On the other hand, natural products provide tremendous opportunities to discover novel therapeutic agents to replace synthetic drugs; thus, research has focused on ethnopharmacognosy. 
The medicinal plant, Eclipta alba (L.) Hassk (E. alba) has been reported to exert numerous therapeutic effects, such as as antitumor (14), anti-hepatic (hepatitics C virus) (15), and anticancer effects (16). It is an excellent source of secondary metabolites, such as flavonoids, phytosterols and coumestans. Phytochemical coumestans, including wedelolactone, demethylwedelolactone and saponins are responsible for the main medicinal effects of E. alba. This medical herb has been reported to posses hair growth-promoting activities, and wedelolactone and demethylwedelolactone have been identified as the major molecules $(17,18)$.

In the present study, we investigated the effects of different extracts of this medicinal plant on nude mouse skin with inherited hair follicular abnormalities. The unique findings may provide new insight for better control of hair loss and demonstrate the roles of the major regulating molecules in the development of nude mouse HFs.

\section{Materials and methods}

Plant sample, extraction and fractionation. Dried aerial parts of $E$. alba were collected from the Jecheon Medicinal Herb Association, Korea and authenticated by Dr Ki Hwan Bae (College of Pharmacy, Chungnam National University, Daejeon, Korea) where the voucher specimens were deposited. The sample was ground into powder and extracted 3 times with petroleum ether at $40^{\circ} \mathrm{C}$ for $4 \mathrm{~h}$ under reflux, then filtered and concentrated under a vacuum evaporator (Serial No. 41440910; EYELA, N-N Series, Rikakikai Co. Ltd. Tokyo, Japan) to yield the corresponding petroleum ether extract (PEE; yield $0.89 \% \mathrm{w} / \mathrm{w}$ ). The resulting residue was then extracted 3 times with methanol at $70^{\circ} \mathrm{C}$ for $4 \mathrm{~h}$ then filtered and evaporated. The dried $\mathrm{MeOH}$ extract residue was suspended in distilled water and the resulting aqueous suspension was fractionated sequentially with the hexane fraction $(\mathrm{HeF})$ and n-butanol fraction $(\mathrm{BuF})$ at 1:1 (v/v) ratio 3 times at room temperature. The resulting 2 fractions and remaining water fraction $(\mathrm{WaF})$ were evaporated under a vacuum (extraction yield, HeF $6.19 \%$, BuF $1.12 \%$ and $\mathrm{WaF} 6.74 \% \mathrm{w} / \mathrm{w})$.

Experimental animals. Athymic male nude (nu/nu) mice of BALB/c origin at 7 weeks of age, were purchased from Dae-Han Biolink, Inc. (Eumseong, Korea). They were kept in autoclaved cages with filter bonnets in a laminar flow unit under $12 \mathrm{~h}$ light:dark periods at $24 \pm 2^{\circ} \mathrm{C}$ in a humidified atmosphere and were fed sterilized food and distilled water. The experiments were performed in the Animal Center of Chungnam National University under aseptic conditions in accordance with the NIH guidelines for the care and use of laboratory animals. The authorization code number is CNU-00244 (Chungnam National University).

Administration of PEE and fractions of E. alba. The mice were divided in to 6 groups; 5 males were allocated to each of the 6 groups. The animals in group 1 received $0.4 \mathrm{ml}$ of the vehicle mixture (propylene glycol:ethanol:dimethyl sulfoxide, 67:30:3\% v/v) (Sigma, St. Louis, MO, USA), and the animals in group 2 received minoxidil 2\% (Mino). The animals in groups 3, 4, 5 and 6 received a $5 \mathrm{mg}$ sample of $\mathrm{PEE}, \mathrm{HeF}, \mathrm{BuF}$ and WaF of E. alba with the vehicle formulation. Treatment
Table I. Scale for the evaluation of hair coverage area in BALB/c athymic nude mice.

\begin{tabular}{lc}
\hline Explanation & Scale \\
\hline Skin pink, no hair & 0 \\
Skin thick, no hair & 1 \\
Skin thick scattered hair & 2 \\
Hair 1-10\% & 3 \\
Hair $10-25 \%$ & 4 \\
Hair $25-50 \%$ & 5 \\
Hair 50-75\% & 6 \\
Hair >75\% & 7 \\
Full body coat & 8 \\
\hline
\end{tabular}

was performed by topical application once per day on the backs of nude mouse skin for 20 consecutive days.

Evaluation of hair coverage area and density. The mice were evaluated for hair coverage area by a score of 0 to 8 as described in Table I. Hair scores were taken on day 0, 5, 7, 12, 16 and 20. To evaluate the change in hair density, digital images of each mouse were randomly acquired on experimental days 8 and 16 in the same region $\left(3.6 \mathrm{~mm}^{2}\right)$ of interscapular skin. The change in hair density was evaluated by analyzing the images (x200 magnification; actual area, $3.6 \mathrm{~mm}^{2}$ ) using Kong, Bom-Viewer Plus software (Bomtech Electronics Co., Ltd., Seoul, Korea).

Histological assessment of hair growth. Skin samples were fixed in $10 \%$ neutral buffered formalin for histological analysis. Paraffin-embedded $4 \mu \mathrm{m}$ sections were stained with Mayer's hematoxylin and eosin (H\&E; Sigma). The morphology and structure of the HFs of the nude mice were evaluated microscopically in the H\&E-stained sections of dorsal skin at a magnification of $x 1,000$. Five fields per section (magnification, x100) were used for counting the number of dermal and subcutaneous HFs with respect to the total number of HFs. Histological processing and digital photomicrographs were acquired using the Leica application suite, version 4.0.0 (Leica Microsystems Ltd., Heerbrugg, Switzerland).

Assessment of keratinocyte proliferation with anti-BrdU. Keratinocyte proliferation was evaluated by an intraperitoneal injection of BrdU (50 mg/kg body weight; Sigma) $1 \mathrm{~h}$ before the mice were sacrificed. Dorsal skin from both the treated and control animals was collected on experimental day 16 and fixed with $4 \%$ paraformaldehyde, dehydrated and embedded in paraffin. After sectioning, the sections were dewaxed and denatured in $1.5 \mathrm{~mol} / 1$ of $\mathrm{HCl}$ for $30 \mathrm{~min}$ and neutralized with phosphate-buffered saline for $1 \mathrm{~h}$. BrdU incorporation was detected by immunohistochemical (IHC) staining of the paraffin-embedded sections with mouse anti-BrdU primary antibody (1:200; Cat. no. SC-32323) in a moist chamber, at room temperature for $3 \mathrm{~h}$ (Santa Cruz Biotechnology, Inc., Santa Cruz, CA, USA). After washing 3 times, the sections were incubated with secondary antibodies [Biotinylated 

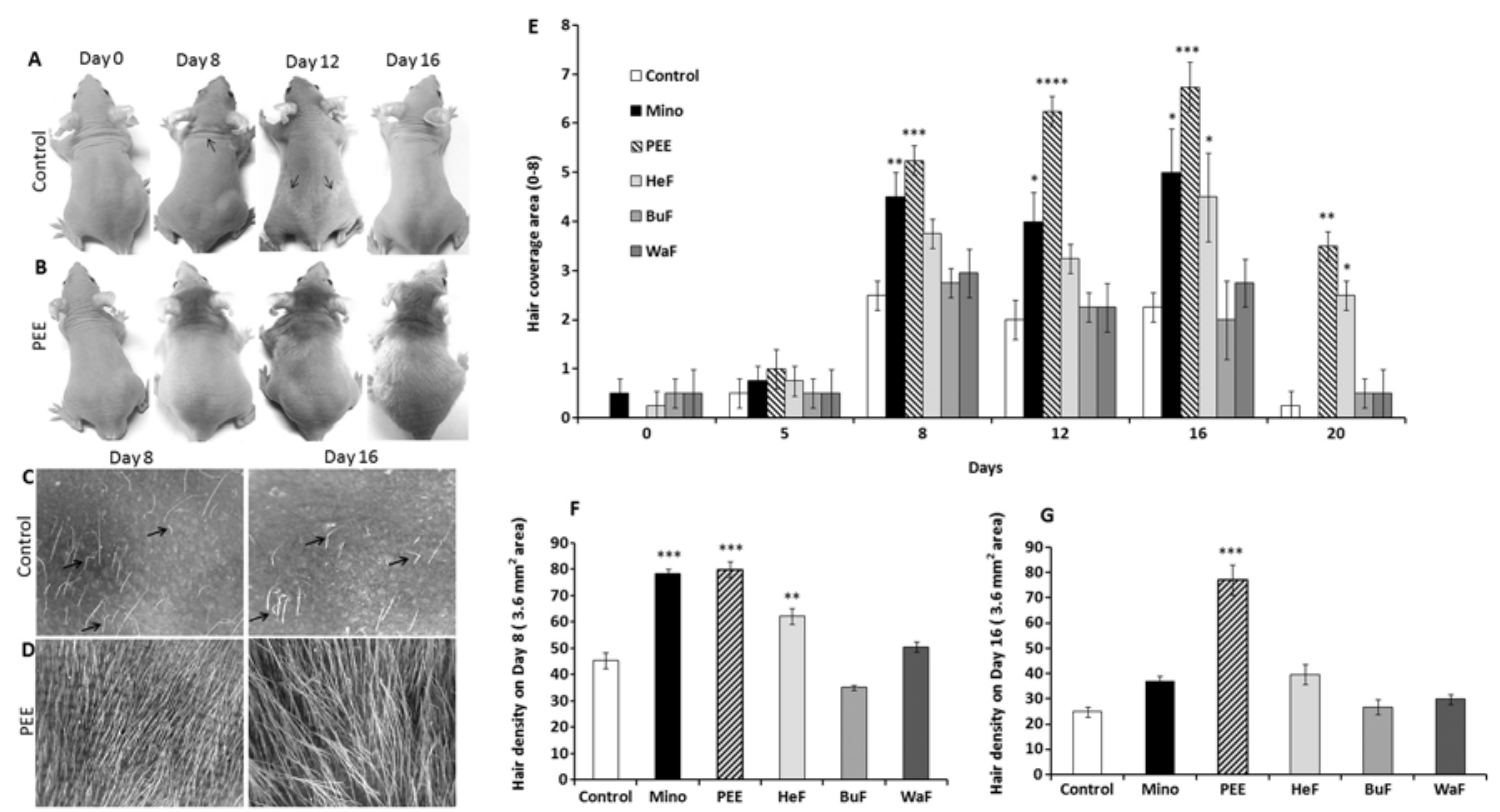

Figure 1. Stimulatory effects of petroleum ether extract (PEE) of E. alba on hair growth in nude mice. (A and E) Vehicle (control)-treated nude mouse skins on days $0,8,12$ and 16 . The vehicle-treated mouse skins exhibited abortive boundaries of short and sparse hair (arrows) on days 8 and 12 and almost complete alopecia was observed on day 16. (B and E) PEE-treated nude mouse skins on days 0,8,12 and 16. The PEE-treated mice showed a consistent increase in hair growth from days 8-16 and exhibited dense and full hair coverage on day 16. (C, F and G) In the contol mice, the few and irregular hair shafts which had emerged on the skin surface began to bend on days 8 and 16. The PEE-treated mouse skins showed noticeably normal, straight hair shafts on day 8 (D and F) which extended up to day 16 (D and G). Digital images were acquired on days 8 and 16 from the skin surface of the (C) vehicle- and (D) PEE-treated nude mice using Kong, Bom-Viewer Plus software at $\mathrm{x} 80$ magnification. Data are expressed as the mean values $(\mathrm{n}=5) \pm$ standard error of the mean (SEM). ${ }^{*} \mathrm{p}<0.05,{ }^{* * *} \mathrm{p}<0.01$ and ${ }^{* * * *} \mathrm{p}<0.001$.

secondary antibody (Life Technologies, Carlsbad, CA, USA)] for $15 \mathrm{~min}$. The assessment of follicular and epidermal keratinocyte and sebaceous gland epithelial cell BrdU labeling was performed by an observer blinded to the treatments using the original magnification of $\mathrm{x} 400$.

Western blot analysis for TGF- $\beta 1$. Western blot analysis was performed to evaluate the expression level of TGF- $\beta 1$ during follicular morphogenesis. The skin samples were homogenized and lysed in protein extraction buffer (Pro-Prep; Intron Biotechnology, Inc., Seongnam, Korea). The protein concentration was measured by Bradford assay (Bio-Rad, Boston, MA, USA); equal amounts of protein ( $20 \mathrm{mg} / \mathrm{sample})$ were separated by $15 \%$ sodium dodecyl sulfate-polyacrylamide gel electrophoresis (SDS-PAGE; Mini Protean II; Bio-Rad) and then transferred onto a PVDF membrane (Immobilon). The membrane was blocked for $12 \mathrm{~h}$ at $4^{\circ} \mathrm{C}$ with $5 \%$ skimmed milk (Uppsala, Sweden) in 1X TBS (10 Mm Tris pH 7.5, $100 \mathrm{mM}$ $\mathrm{NaCl}$ and $0.5 \%$ Tween-20). Immunodetection was performed by incubation at appropriate dilutions (1:500, TGF- $\beta 1$ polyclonal antibody) at $4^{\circ} \mathrm{C}$ overnight then incubated with the secondary antibodies goat anti-rabbit IgG-HRP (both from Santa Cruz Biotechnology, Inc.) conjugate for $1 \mathrm{~h}$ at room temperature. After washing, the blots were detected by ECL western blotting detection reagents (Santa Cruz Biotechnology, Inc.). The band intensities were quantified using NIH ImageJ software.

Statistical analysis. The experimental data are expressed as the means \pm standard deviation (SD). The Student's t-test or one-way ANOVA were used for the assessment of significance between the different treatment groups. Statistical analysis was performed using SAS 9.2 software. A value of $p<0.05$ was considered to indicate a statistically significant difference.

\section{Results}

Stimulatory effect of PEE of E. alba on hair growth patterns in nude mice. The changes occurring in hair growth patterns in athymic nude mice were documented after the initiation of topical application for 20 consecutive days. The effects of PEE of E. alba were evident as early as 7 days following the commencement of treatment, and became obvious on the dorsal body surface. Hair growth on the PEE-treated mice first became evidient on the heads then consistently extended to the caudal region of the tail on experimental day 16 (Fig. 1B). On the other hand, the control mice exhibited relatively sparse and shorter hair growth that was roughly distributed on different regions of the body on day 10; this phenomena noticeably corresponds to the 'wave like pattern' of the nude phenotype (Fig. 1A) (19). Eventually, the control mice became nearly complete 'nude' on experimental day 16 (Fig. 1A and C), while PEE induced a distinctly smoother, thicker hair shaft, leading to dense and marked hair coverage on days 8 and 16 (Fig. 1B and D).

Effects of PEE of E. alba on hair coverage area and density. The stimulatory effects of PEE of E. alba on the hair coverage area of the nude mice were evaluated as they received specific concentrations of PEE, $\mathrm{HeF}, \mathrm{BuF}, \mathrm{WaF}$ vs. the vehicle and or $2 \%$ minoxidil. The effects of PEE on the hair coverage area of the mice in all treatment groups were precisely estimated for each mouse by giving them a score from 0 to 8 (Table I). 


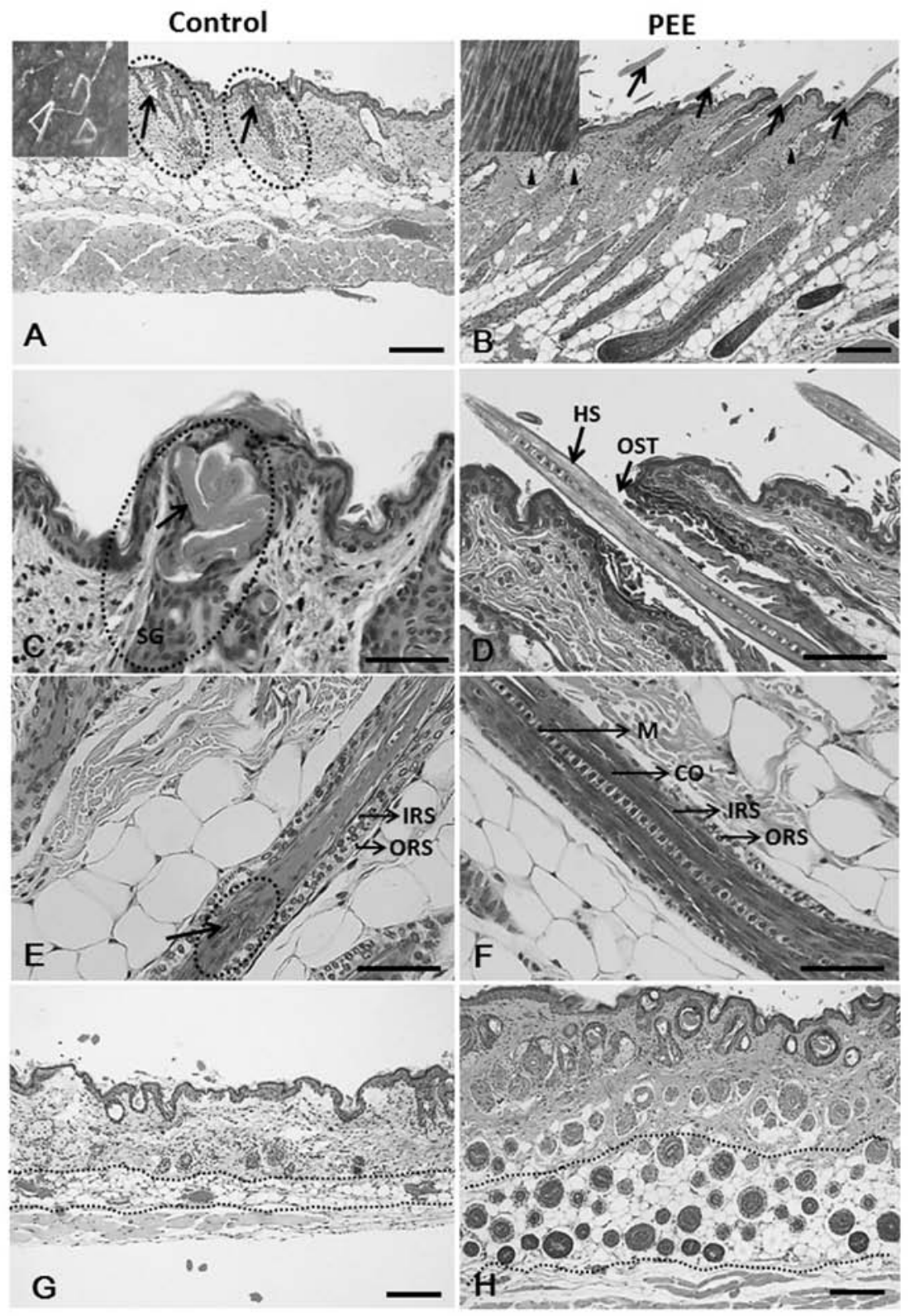

Figure 2. Hematoxylin and eosin (H\&E)-stained skin sections of the vehicle- and petroleum ether extract (PEE)-treated mice. (A, arrows and inset) Fragmented hair shafts with keratinized debris that do penetrate the epidermis are heavily twisted. Note that vehicle-treated nude mouse HFs reveal histological signs of the late catagen phase on day 16. (B) PEE-treated mouse skins exhibit prominent follicles (arrows), as well as moderate sebaceous gland hypertrophy (arrowheads) and the presence of HFs above the skin surface (arrows and inset). Note that the HFs of the PEE-treated mice were uniformly in the late anagen phase of the hair cycle. (C) Hair shaft twist and coil at the level of sebaceous gland in the vehicle-treated mice skin specimen. (D) PEE-treated mouse skin specimen shows straight hair shaft penetrating the skin surfac. (E) Vehicle-treated nude follicle, cortex formation is severely injured. (F) Hair shaft regularly formed, intact and coated by a clearly discernible hair cuticle in PEE-treated mice. (G) Control mouse specimen with abortive HFs (arrows) and lower number of HFs. (H) PEE-treated nude mice reveal normal HFs and an increase in the number of hair follicles. HS, hair shaft; IRS, inner root sheath; ORS, outer root sheath; M, medulla; CO, cortex; SG, sebaceous gland; HF, hair follicle; OST, ostium. Scale bars: (A, B, G and H) $100 \mu \mathrm{m}$; (C-F) $50 \mu \mathrm{m}$.

The maximum hair growth score was significantly $(\mathrm{p}<0.001)$ increased in the mice treated with PEE of E. alba than in the mice in the other groups from day 8 and consistently covered the maximum area of the body on day 16 (Fig. 1E). By contrast, in the nude mice, rapid hair loss was observed in the minoxidil and other treatment groups, which then returned to baseline levels. In terms of hair density, on days 8 and 16 following the initiation of treatment, the mice in the PEE-treated group exhibited a significant increase $(\mathrm{p}<0.0001)$ in hair density compared to the other groups (Fig. 1G). Although, minoxidil had a significant effect $(\mathrm{p}<0.001)$ on sustaining hair density on day 8 , progressive hair loss decreased and hair density thus also decreased on day 16. This is a characteristic pattern observed in the hair growth patterns of athymic nude mice.

Stimulatory effects of PEE of E. alba on distorted HFs of athymic nude mice. The skin specimens of the control mice exhibited numerous dystrophic HFs, that twisted and coiled within the follicular infundibulum (Fig. 2C) and failed to penetrate the epidermis. However, the hair shafts that penetrated the epidermis were heavily twisted and frequently fractured before achieving a substantial length (Fig. 2A), as is normally evident 


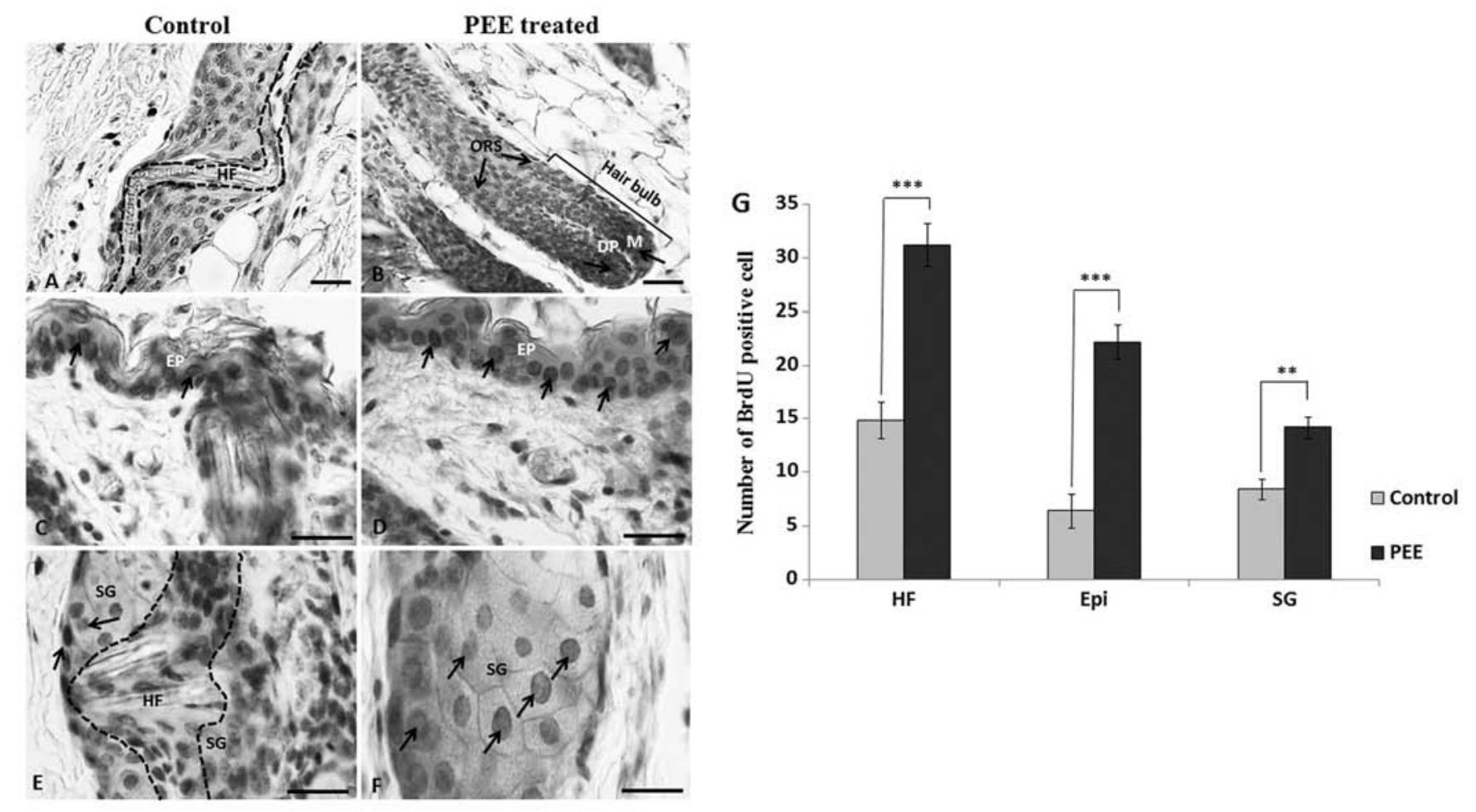

Figure 3. BrdU immunohistochemistry for analysis of keratinocyte proliferation in the vehicle- and petroleum ether extract (PEE)-treated mouse skins. (A) Abortive HFs of mice treated with the vehicle. (B) Arrows indicate BrdU-labeled cells in both the hair matrix and outer root sheath of mice treated with PEE. (C) Only a small number of labeled cells was observed in the control group. (D and G) Note the increase in the number of BrdU-positive proliferating epidermal keratinocytes in the PEE-treated group compared to the control mice. (E and G) Hypertrophic sebaceous gland with distorted HF (dotted line) containing BrdU labeled epithelial cell. Note the increase in the number of BrdU-labeled epithelial cells in the sebaceous gland (arrows) in the PEE-treated nude mice compared with the control mice. Scale bar: (A and B) $100 \mu \mathrm{m},(\mathrm{C}-\mathrm{F}) 50 \mu \mathrm{m}$. M, matrix; DP, dermal papilla; HF, hair follicle; EP, epidermis; SG, sebaceous gland. Data are presented as the means \pm SD of 3 independent experiments; ${ }^{* *} \mathrm{p}<0.01$ and ${ }^{* * *} \mathrm{p}<0.001$ vs. control.

in nude mouse skin. This striking characteristic of nude mice indicates that the hair fibers suffer from abnormal keratinization (20). On the other hand, the skins of the PEE-treated nude mice had relatively normal follicles containing well differentiated straight hair shafts which continued through the follicular ostia to the skin's surface (Fig. 2B and D). In nude mice, the structure of the HFs, inner root sheath and hair shaft exhibit abnormalities (8), the most striking of which is the cuticle of the HF which is either discontinuous or, more often, totally absent (Fig. 2E). By contrast, the HFs of the PEE-treated mice were regularly formed and intact, coated by a clearly discernible hair cuticle (Fig. 2F). Furthermore, the control mice exhibited abortive HFs which revealed histological signs of the late catagen stage (Fig. 2A and G). On the other hand, treatment with PEE of E. alba resulted in a histological pattern identical to that observed in the late anagen phase of cycling hair and induced a marked increase in the number of HFs compared with the control mice (Fig. 2H).

PEE of E. alba enhances keratinocyte proliferation in the follicular matrix. To determine the difference in the follicular keratinocyte proliferation rate, we labeled the proliferating cells with $\mathrm{BrdU}$ in vivo on day 16 and subsequently stained them with an antibody to BrdU. Our results revealed that in the PEE-treated mice, the number of BrdU-labeled keratinocytes per anagen follicle increased significantly, particularly in the follicular matrix and outer root sheath compared to the control mice (Fig. 3A and B). The mean number of BrdU-positive cells per anagen follicle was $31.2 \pm 2$ in the PEE-treated mice as compared with $14.9 \pm 1.7$ in the control mice. This increase was statistically significant $(p<0.001)$. Moreover, the PEE-treated nude mice also exhibited a significant increase in the number of BrdU-labeled proliferating epidermal keratinocytes $(\mathrm{p}<0.001)$ and BrdU-positive epithelial cells per sebaceous gland $(\mathrm{p}<0.01)$ (Fig. 3D, F and G).

Expression of TGF- $\beta 1$ during follicular morphogenesis. The expression level of TGF- $\beta 1$ was quantified by western blot analysis at different time point (on days 8 and 16) to determine the comparative expression of TGF- $\beta 1$ in the vehicle- and PEE-treated mouse skins. Quantitative analysis of the western blots revealed a significant $(\mathrm{p}<0.001)$ decrease in the expression levels of TGF- $\beta 1$ in the PEE-treated mice during early anagen (day 8) and during the late anagen or anagen-catagen transition (day 16) compared with the control mice (Fig. 4). Quantitative analysis indicated that the delayed hair regression in the PEE-treated mouse skins was associated with an altered expression of TGF- $\beta 1$.

\section{Discussion}

In this study, we investigated the hair growth stimulatory effects of PEE. Treatment involved the topical application of PEE and different solvent fractions of $E$. alba on the skins of nude mice. 

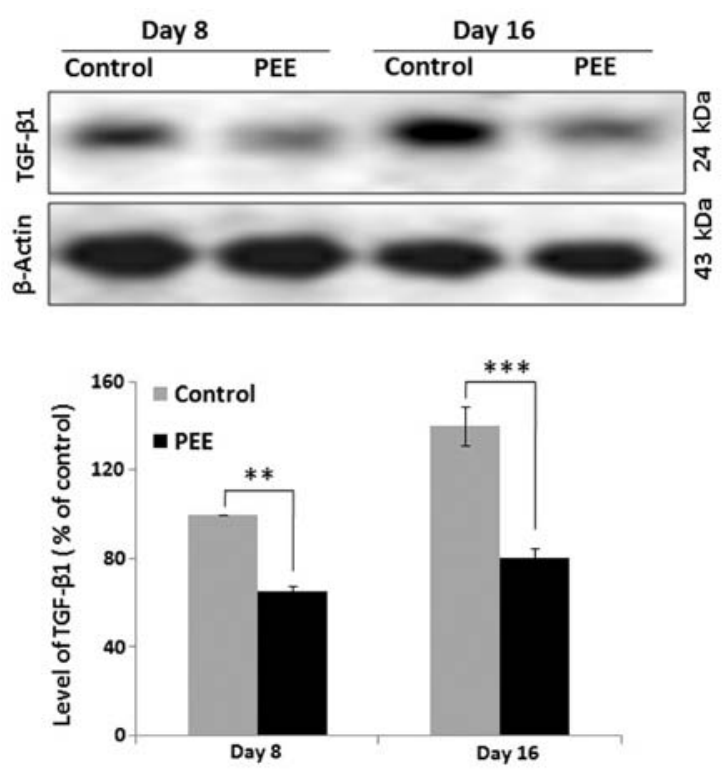

Figure 4. Transcriptional growth factor- $\beta 1$ (TGF- $\beta 1$ ) protein expression in the vehicle- and petroleum ether extract (PEE) ( $5 \mathrm{mg} / \mathrm{day})$-treated nude mice on days 8 and 16 following the initiation of treatment. Quantification of the relative expression of TGF- $\beta 1$ showed significantly reduced levels of total TGF- $\beta 1$ during the early anagen phase (day 8 ) and the late anagen or anagen-catagen transition (day 16) in PEE-treated mice ( $\mathrm{n}=3$; Student's t-test; ${ }^{* *} \mathrm{p}<0.05$ and **** $\mathrm{p}<0.001$; error bars represent the means $\pm \mathrm{SD})$.

Among the treatment groups, PEE had an outstanding effect on hair growth in the nude mice. In the PEE-treated nude mice, the skin surface exhibited a large number of HFs penetrating the epidermis. This evidence, together with previous data has raised the possibility that PEE of E. alba may have profound effects on HFs in nude mice (21). This unique finding also establishes the hypothesis that nude mice are not hairless and that the development and differentiation of HFs are injured by severe disturbances of the keratinization process $(8,21,22)$.

Normal hair growth requires a balance between keratinocyte growth and differentiation in the HF (23). However, in nude mice, the keratinization processes is markedly impaired, and as a result, HFs in nude mice exhibit structural abnormalities in the cortex and inner root sheath (8). The results from our histological specimens revealed that PEE of E. alba may modulate the structural defects of HFs in nude mice. Moreover, IHC staining also revealed that PEE of E. alba stimulated follicular proliferation in hair matrix cells and induced to neutralized defects in nude epidermal keratin differentiation. Therefore, it has a directly effect on HFs in nude mice by compensating for inherent genetic defects.

The anagen-to-catagen transition is known to be driven by factors, such as TGF- $\beta 1$ and TGF- $\beta 2$ and characterized by apoptotic cell death in hair bulb epithelial cells and outer root sheath (ORS) cells $(5,24-28)$. In our study, the topical application of PEE of $E$. alba led to a decrease in TGF- $\beta 1$ expression in nude mice, leading to enhanced keratinocyte proliferation and thereby prolonging the anagen stage in the PEE-treated mice. On the other hand, the vehicle-treated mice exhibited premature catagen development and a reduced number of proliferating keratinocytes in the HFs. Notably, the normal expression of TGF- $\beta 1$ controls follicular regression in both mice and humans in vivo $(5,27)$. More importantly, the high expression of TGF- $\beta 1$ in the epidermis leads to the suppression of epithelial cell proliferation and the eventual inhibition of normal skin development (4). Thus, the alteration of TGF- $\beta 1$ signaling in PEE-treated mice may be associated with the enhanced keratinocyte proliferation and subsequently delayed hair regression phase.

In conclusion, the present study demonstrates the precise biological mechanisms and underlying effects of PEE of E. alba on hair growth, as well as the anti-apoptotic effects of this standardized extract. Thus, PEE of E. alba may be considered an effective standardized extract that modulates defects in keratinocyte differentiation in the HFs of nude mice by promoting the proliferation of epidermal basal cells and cells in the hair matrix. Based on these fruitful findings, the use of such a stimulatory agent may provide a novel strategy for the management of various forms of alopecia and may have clinical implications for hair loss.

\section{References}

1. Paus R: Control of the hair cycle and hair diseases as cycling disorders. Curr Opin Dermatol 3: 248-258, 1996.

2. Paus R: Principles of hair cycle control. J Dermatol 25: 793-802, 1998.

3. Rishikaysh P, Dev K, Diaz D, Qureshi WM, Filip S and Mokry J: Signaling involved in hair follicle morphogenesis and development. Int J Mol Sci 15: 1647-1670, 2014.

4. Sellheyer K, Bickenbach JR, Rothnagel JA, Bundman D, Longley MA, Krieg T, Roche NS, Roberts AB and Roop DR: Inhibition of skin development by over expression of transforming growth factor beta 1 in the epidermis of transgenic mice. Proc Natl Acad Sci USA 90: 5237-5241, 1993.

5. Foitzik K, Lindner G, Mueller-Roever S, Maurer M, Botchkareva N, Botchkarev V, Handjiski B, Metz M, Hibino T, Soma T, Dotto GP and Paus R: Control of murine hair follicle regression (catagen) by TGF-beta1 in vivo. FASEB J 14: 752-760, 2000.

6. Flanagan SP: 'Nude' a new hairless gene with pleiotropic effects in the mouse. Genet Res 8: 295-309, 1966.

7. Pantelouris EM: Athymic development in the mouse. Differentiation 1: 437-450, 1973.

8. Köpf-Maier P, Mboneko VF and Merker HJ: Nude mice are not hairless. A morphological study. Acta Anat (Basel) 139: 178-190, 1990.

9. Mecklenburg L, Nakamura M, Sundberg JP and Paus R: The nude mouse skin phenotype: the role of Foxn1 in hair follicle development and cycling. Exp Mol Pathol 71: 171-178, 2001.

10. Mecklenburg L, Tychsen B and Paus R: Learning from nudity: lessons from the nude phenotype. Exp Dermatol 14: 797-810, 2005.

11. Gafter-Gvili A, SredniB, Gal R, Gafter U and Kalechman Y: Cyclosporin A-induced hair growth in mice is associated with inhibition of calcineurin-dependent activation of NFAT in follicular keratinocytes. Am J Physiol Cell Physiol 284: C1593-C1603, 2003

12. Danilenko DM, Ring BD, Yanagihara D, Benson W, Wiemann B, Starnes CO and Pierce GF: Keratinocyte growth factor is an important endogenous mediator of hair follicle growth, development, and differentiation. Normalization of the nu/nu follicular differentiation defect and amelioration of chemotherapy-induced alopecia. Am J Pathol 147: 145-154, 1995.

13. Sredni B, Gal R, Cohen IJ, Dazard JE, Givol D, Gafter U, Motro B, Eliyahu S, Albeck M, Lander HM and Kalechman Y: Hair growth induction by the Tellurium immunomodulator AS101: association with delayed terminal differentiation of follicular keratinocytes and ras-dependent up-regulation of KGF expression. FASEB J 18: 400-402, 2004.

14. Liu QM, Zhao HY, Zhong XK and Jiang JG: Eclipta prostrata L. phytochemicals: isolation, structure elucidation, and their antitumor activity. Food Chem Toxicol 50: 4016-4022, 2012.

15. Manvar D, Mishra M, Kumar S and Pandey VN: Identification and evaluation of anti Hepatitis $\mathrm{C}$ virus phytochemicals from Eclipta alba. J Ethnopharmacol 144: 545-554, 2012. 
16. Chaudhary H, Dhuna V, Singh J, Kamboj SS and Seshadri S: Evaluation of hydro-alcoholic extract of Eclipta alba for its anticancer potential: an in vitro study. J Ethnopharmacol 136 : 363-367, 2011

17. Roy RK, Thakur M and Dixit VK: Hair growth promoting activity of Eclipta alba in male albino rats. Arch Dermatol Res 300: 357-364, 2008.

18. Datta K, Singh AT, Mukherjee A, Bhat B, Ramesh B and Burman AC: Eclipta alba extract with potential for hair growth promoting activity. J Ethnopharmacol 124: 450-456, 2009.

19. Militzer K: Hair Growth pattern in nude mice. Cells Tissues Organs 168: 285-294, 2001.

20. Schlake T, Schorpp M, Maul-Pavicic A, Malashenko AM and Boehm T: Forkhead/winged-helix transcription factor Whn regulates hair keratin gene expression: molecular analysis of the nude skin phenotype. Dev Dyn 217: 368-376, 2000.

21. Hozumi Y, Imaizumi T and Kondo S: Effect of cyclosporin on hair-existing area of nude mice. J Dermatol Sci 7 (Suppl): S33-S38, 1994

22. Panteleyev AA, Paus R, Ahmad W, Sundberg JP and Christiano AM: Molecular and functional aspects of the hairless (hr) gene in laboratory rodents and humans. Exp Dermatol 7: 249-267, 1998
23. Fuchs E: Epidermal differentiation and keratin gene expression. J Cell Sci Suppl 17: 197-208, 1993.

24. Paus R, Foitzik K, Welker P, Bulfone-Paus S and Eichmüller S: Transforming growth factor-beta receptor type I and type II expression during murine hair follicle development and cycling. J Invest Dermatol 109: 518-526, 1997.

25. Hardy MH: The secret life of the hair follicle. Trends Genet 8 : 55-61, 1992.

26. Soma T, Dohrmann CE, Hibino T and Raftery LA: Profile of transforming growth factor-beta responses during the murine hair cycle. J Invest Dermatol 121: 969-975, 2003.

27. Soma T, Tsuji Y and Hibino T: Involvement of transforming growth factor beta2 in catagen induction during the human hair cycle. J Invest Dermatol 118: 993-997, 2002.

28. Tsuji Y, Denda S, Soma T, Raftery L, Momoi T and Hibino T: A potential suppressor of TGF-beta delays catagen progression in hair follicles. J Investig Dermatol Symp Proc 8: 65-68, 2003. 